\title{
The ideals of the hurwitzean polynomial ring
}

\section{Margaret J. Morton}

In 1919, Adolf Hurwitz formed the quaternion ring $R$ composed of elements whose coordinates were either all integers or halves of odd integers. The objective of this paper is to examine the (two-sided) ideal structure in the hurwitzean polynomial ring $R[x]$, formed by taking all polynomials with coefficients in $R$. The maximal and prime ideals of $R[x]$ will be characterized with results surprisingly analogous to those in $Z[x]$. In addition, a canonical basis, of the type developed by G. Szekeres, 1952, for polynomial domains, will be developed for the ideals of $R[x]$.

\section{A. Preliminaries}

The hurwitzean ring of quaternions $(R)$ is formed of all quaternions $\alpha=a_{0}+a_{1} i+a_{2} j+a_{3} k$ where

(i) the coordinates $a_{0}, a_{1}, a_{2}, a_{3}$ are either all integers or are all halves of odd integers,

(ii) the units $i, j, k$ satisfy the relations $i^{2}=j^{2}=k^{2}=-1, \quad i j=k=-j i, \quad j k=i=-k j, \quad k i=j=-i k$. The conjugate of $\alpha$ is $\bar{\alpha}=a_{0}-a_{1} i-a_{2} j-a_{3} k$. The norm of $\alpha$ is $N(\alpha)=\alpha \bar{\alpha}=\bar{\alpha} \alpha=a_{0}^{2}+a_{1}^{2}+a_{2}^{2}+a_{3}^{2}$. For all $\alpha$ and $\beta$ in $R, N(\alpha)$ is in $Z$ and $N(\alpha \beta)=N(\alpha) N(\beta)$. The trace of $\alpha$ is $\operatorname{tr}(\alpha)=\alpha+\bar{\alpha}$. Received 29 October 1975. 
$\operatorname{tr}(\alpha)$ is in $Z$ for all $\alpha$ in $R \cdot R$ is the maximal quaternion ring with the property that if $\alpha$ is in $R$, then $N(\alpha)$ and $\operatorname{tr}(\alpha)$ are in Z.

If $\alpha$ is in $R$, then $\alpha$ is a unit, if and only if $N(\alpha)=1$. The group of units of $R$ consists of the twenty-four quaternions $\pm 1, \pm i, \pm j$, $\pm k, \frac{1}{2}( \pm 1 \pm i \pm j \pm k)$.

The center of $R$ is $Z$. Closely related are elements in $R$ of norm two. Any such element which is a right divisor of an element in $R$ is also a left divisor and vice versa.

Rédei [2] showed:

THEOREM 1. AZZ the distinct ideals of $R$, different from zero, are the principal ideals $\left(m \lambda^{t}\right)$, where $m=1,2, \ldots, t=0,1$, $\lambda=1+i$.

From this theorem it follows quite readily that all ideals in $R$ generated by elements of norm two are equal and that all ideals in $R$ commute. The ideals of $R$ will be denoted by $A, B, C, \ldots$.

It can also be shown that:

THEOREM 2. The following are equivalent:

(i) $P$ is a proper prime ideal in $R$;

(ii) $P$ is a proper maximal ideal in $R$;

(iii) $P=(p)$, where $p \neq 1$ is an odd prime in 2 , or $P=(\lambda)$.

Let $K[x]$ be the quaternion polynomial ring composed of all elements $\rho(x)=r_{0}(x)+r_{1}(x) i+r_{2}(x) j+r_{3}(x) k$, where $r_{0}(x), r_{1}(x), r_{2}(x), r_{3}(x)$ are in $Q[x]$. Then $K[x]$ is a non-commutative integral domain with the obvious multiplication and addition. For an element $\rho(x)$ in $K[x]$, conjugate, norm and trace are defined as in $R$. In addition the symbol $\partial$ will be used to denote the degree of a polynomial. For any elements

$$
\rho(x)=r_{0}(x)+r_{1}(x) i+r_{2}(x) j+r_{3}(x) k
$$

and

$$
\tau(x)=t_{0}(x)+t_{1}(x) i+t_{2}(x) j+t_{3}(x) k
$$


in $K[x]$ the following results are easily verified.

(i) If $q(x)$ is in $Q[x]$, then $q(x) \rho(x)=\rho(x) q(x)$ (that is, $Q[x]$ is the center of $K[x])$.

(ii) $N(\rho(x) \tau(x))=N(\rho(x)) N(\tau(x))$.

(iii) $\partial N(\rho(x) \tau(x))=\partial N(\rho(x))+\partial N(\tau(x))$.

(iv) $\partial N(\rho(x)+\tau(x)) \leq \max \{\partial N(\rho(x)), \partial N(\tau(x))\}$.

(v) $\partial N(\rho(x))=0$, if and only if, $r_{0}(x), \ldots, r_{3}(x)$ are in $Q$. Such elements $\rho(x)$ are in the quaternion ring.

DEFINITION. $\rho(x)$ is a unit in $K[x]$ if there exists $\sigma(x)$ in $K[x]$ such that either $\rho(x) \sigma(x)=1$ or $\sigma(x) \rho(x)=1$.

It is not necessary to distinguish between left and right units in $K[x]$. For if $\rho(x) \sigma(x)=1$, then $\overline{\rho(x)}=\overline{\rho(x)} \rho(x) \sigma(x)=\sigma(x) \rho(x) \overline{\rho(x)}$, so $1=\sigma(x) \rho(x)$.

THEOREM 3 (Division Algorithm). Given $\rho(x)$ and $\sigma(x)$ not units in $K[x]$, there exist $\tau(x)$ and $\mu(x)$ in $K[x]$ such that $\rho(x)=\tau(x) \sigma(x)+\mu(x)$, where $\partial \mu(x)<\partial \sigma(x)$. (As stated this is a right division algorithm. Similarly, there is a left division algorithm.)

THEOREM 4 (Existence of a greatest common divisor). Any two elements $\rho(x)$ and $\sigma(x)$ in $K[x]$, which are not both zero, have $a$ greatest common right divisor $\phi(x)$ which is uniquely determined up to a unit.

Furthermore, there exist $\psi(x)$ and $\omega(x)$ in $K[x]$ such that $\phi(x)=\rho(x) \psi(x)+\sigma(x) \omega(x)$. (A similar result holds for a greatest common left divisor.)

DEFINITION. Let $p(x)=r_{0}(x)+r_{1}(x) i+r_{2}(x) j+r_{3}(x) k$ be in $K[x]$. Then $\rho(x)$ is primitive in $K[x]$ if the greatest common divisor of $r_{0}(x), \ldots, r_{3}(x)$ in $Q[x]$ is a unit.

The ideals of $K[x]$ will be denoted by $S(x), T(x), \ldots$.

THEOREM 5. AlZ the distinct ideals of $K[x]$, different from zero, are the principal ideals $(a(x))$, where $a(x)$ is in $2[x]$.

Proof. It follows from Theorem 3 that $K[x]$ is a principal ideal 
domain.

$$
\text { Let } \begin{aligned}
S(x)= & (\sigma(x)) \text { be an ideal in } K[x] \text { where } \\
& \sigma(x)=s_{0}(x)+s_{1}(x) i+s_{2}(x) j+s_{3}(x) k
\end{aligned}
$$

is a primitive element in $K[x]$. Then

$$
i \sigma(x) i+j \sigma(x) j+k \sigma(x) k=-4 s_{0}(x)+\sigma(x),
$$

so $4 s_{0}(x)$ is in $S(x)$. Furthermore,

$$
2(i \sigma(x) j-j \sigma(x) i)=4 s_{3}(x)+4 s_{0}(x),
$$

hence $4 s_{3}(x)$ is in $S(x)$. Similar calculations show that $4 s_{1}(x)$ and $4 s_{2}(x)$ are in $S(x)$. But $\sigma(x)$ is primitive, so the greatest common divisor in $Q[x]$ of $4 s_{0}(x), \ldots, 4 s_{3}(x)$ must be a unit. By Theorem 4 this greatest common divisor must be in $S(x)$. Hence $S(x)$ contains a unit and must equal $K[x]$.

Let $T(x)$ be any proper ideal in $K[x]$. Then $T(x)=(\tau(x))$, where $\tau(x)$ is a nonprimitive element in $K[x]$. Let $\tau(x)=q(x) \sigma(x)$, where $q(x)$ is in $Q[x]$ and $\sigma(x)$ is primitive in $K[x]$. Then,

$$
T(x)=(\tau(x))=(q(x))(\sigma(x))=(q(x)) .
$$

Let $l$ be the lowest common multiple of the denominators of $q(x)$, then $q(x)=Z^{-1} a(x)$, where $a(x)$ is in $z[x]$. Since $z$ is a unit in $K[x]$ it now follows that $T(x)=(a(x))$.

THEOREM 6. The following are equivalent:

(i) $M(x)$ is a proper maximal ideal in $K[x]$;

(ii) $M(x)$ is a proper prime ideal in $K[x]$;

(iii) $M(x)=(p(x))$, where $\partial p(x) \geq 1$ and $p(x)$ is irreducible in $Z[x]$.

\section{B. The quaternion factor rings $R_{\lambda}[x]$ and $R_{p}[x]$}

Before the quaternion polynomial ring $R[x]$ can be discussed it is necessary to examine the structure of certain quaternion factor rings. 
Let $\lambda=1+i$ and $p$ be an odd prime in $z$. Then $R_{\lambda}=\frac{R}{(\lambda)}$, $R_{\lambda}[x]=\frac{R}{(\lambda)}[x], R_{p}=\frac{R}{(p)}$, and $R_{p}[x]=\frac{R}{(p)}[x]$ are all quaternion factor rings.

$R_{\lambda}$ is a finite field with four elements. It has a complete set of representatives, namely $0,1, \frac{1}{2}(1+i+j+k)$ and $\frac{1}{2}(1-i-j-k)$, in $R$. Thus $R_{\lambda}[x]$ is a commutative principal ideal domain with a complete set of representatives in $R[x]$. By the same type of proof used for $z[x]$ it follows that the proper maximal and prime ideals in $R_{\lambda}[x]$ are generated by the irreducible elements of $R_{\lambda}[x]$.

THEOREM 7. (i) $R_{p}$ is isomorphic to the ring of quatemions with coordinates in $Z_{p}$ and consequently has $p^{4}$ elements.

(ii) $R_{p}[x]$ is isomorphic to the ring of quatemions with coordinates in $z_{p}[x]$.

(iii) $R_{p}$ is isomorphic to the full ring of two by two matrices with entries in $Z_{p}$.

(iv) $R_{p}[x]$ is isomorphic to the full ring of two by two matrices with entries in $z_{p}[x]$.

(v) $R_{p}[x]$ is a principal ideal ring.

Proof. (i) Clearly $z_{p} \subseteq R_{p}$. Since $p \neq 2,2^{-1}$ is in $z_{p}$ and the desired result follows.

(ii) Immediate from (i).

(iii) By Theorem 2, $(p)$ is a proper maximal ideal in $R$. By (i), $R_{p}$ has only a finite number of elements, thus it can have only a finite number of maximal ideals and must be simple. Therefore, by the WedderburnArtin structure theorem, $R_{p}$ must be isomorphic to a full matrix ring over a division ring. But by Theorem 1 this full matrix ring must have $p^{4}$ elements, thus the matrices must be two by two. Moreover the division ring 
must contain $p$ elements, so, without loss of generality, it can be taken as $Z_{p}$.

\section{(iv) Follows from (iii).}

(v) Let $A(x)$ be an ideal in $R_{p}[x]$ and $\alpha(x)=\left[\begin{array}{ll}\alpha_{11}(x) & a_{12}(x) \\ \alpha_{21}(x) & a_{22}(x)\end{array}\right]$, where $a_{m n}(x)$ is in $z_{p}[x]$ for $n=1,2, m=1,2$, be any element in $A(x)$. Using the fact that $A(x)$ is a two-sided ideal it follows that the matrices $\left[\begin{array}{cc}a_{m n}(x) & 0 \\ 0 & 0\end{array}\right], n=1,2, m=1,2$, are in $A(x)$. Let $L(x)=\left\{k(x)\right.$ in $z_{p}[x] \mid\left[\begin{array}{cc}k(x) & 0 \\ 0 & 0\end{array}\right]$ in $\left.A(x)\right\}$. Then $L(x)$ is a non-trivial ideal in $z_{p}[x]$. But $z_{p}[x]$ is a principal ideal ring, hence $L(x)=(Z(x))$ for some $Z(x)$ in $L(x)$. Thus $\left(\left[\begin{array}{cc}Z(x) & 0 \\ 0 & 0\end{array}\right]\right)$ is contained in $A(x)$.

Conversely, since $a_{m n}(x), m=1,2, n=1,2$, are in $L(x)$ it follows that in $z_{p}[x], a_{m}(x)=Z(x) b_{m n}(x)$ for $m=1,2, n=1,2$. Thus

$$
\begin{gathered}
\alpha(x)=\left[\begin{array}{cc}
l(x) & 0 \\
0 & l(x)
\end{array}\right]\left[\begin{array}{ll}
b_{11}(x) & b_{12}(x) \\
b_{21}(x) & b_{22}(x)
\end{array}\right], \\
\text { so } A(x) \text { is contained in }\left(\left[\begin{array}{cc}
z(x) & 0 \\
0 & Z(x)
\end{array}\right]\right) .
\end{gathered}
$$

It is clear from this Theorem that $R_{p}$ has a complete set of representatives in $R$ and $R_{p}[x]$ has a complete set of representatives in $R_{p}[x]$.

DEFINITION. Let $\alpha(x)=a_{0}(x)+a_{1}(x) i+a_{2}(x) j+a_{3}(x) k$ be an element in $R_{p}[x]$. Then $\alpha(x)$ is primitive if the greatest common 
aivisor of the $a_{\eta}(x), 0 \leq \ell \leq 3$, in $z_{p}[x]$ is a unit.

THEOREM 8. (i) The only proper ideals in $R_{p}[x]$ are of the form $(a(x))$, where $a(x) \neq 1 \bmod p$ is in $z_{p}[x]$.

(ii) The proper prime and maximal ideals in $R_{p}[x]$ are $(p(x))$, where $p(x) \neq 1 \bmod p$ is irreducible in $z_{p}[x]$.

Proof. (i) This follows by the same type of argument that was used in Theorem 5 .

(ii) By $(i)$ the proper ideals in $R_{p}[x]$ commute, so the desired result follows by the standard method.

\section{The quaternion polynomial ring $R[x]$}

The ring $R[x]$ is clearly a subring of $K[x]$. Thus the definitions made for $K[x]$ are applicable for $R[x]$. However, the structure of $R[x]$ is more complicated than that of $K[x] . R[x]$ does not have a division algorithm and is not a principal ideal domain. It can be verified that it is a noetherian ring. The ideals of $R[x]$ will be denoted by $A(x), B(x), C(x), \cdots$.

In $R$ ideals other than those generated by a unit were equal to the whole ring. The same type of situation arises in $R[x]$ as will be shown in Theorem 9 .

Let $\phi_{\lambda}$ denote the natural epimorphism from $R[x]$ to $R_{\lambda}[x]$, where $\lambda=1+i$. Let $\phi_{p}$ denote the natural epimorphism from $R[x]$ to $R_{p}[x]$, where $p$ is again an odd prime in $Z$.

THEOREM 9. Let $B(x)$ be an ideal in $R[x]$. Then $B(x)=R[x]$, if and only if either

(i) $B(x)=(\alpha(x), p)$, where $\phi_{p}(\alpha(x))$ is primitive in

$$
R_{p}[x] ; \text { or }
$$

(ii) $B(x)=(\alpha(x), \lambda)$, where $\phi_{\lambda}(\alpha(x)) \equiv 1 \bmod \lambda$ in $R_{\lambda}[x]$.

Proof. Case 1: $B(x)$ contains prime $p \neq 2$. Now $(p)$ is in the 
kernel of $\phi_{p}$ and $(p) \subseteq B(x)$, thus $R[x] / B(x) \cong R_{p}[x] / \phi_{p}(B(x))$.

If $R[x]=B(x)$, then $R_{p}[x]=\phi_{p}(B(x))$, so by Theorem 8 , $\phi_{p}(B(x))=\left(\alpha_{p}(x)\right)$, where $\alpha_{p}(x)$ is primitive in $R_{p}[x]$. But $\phi_{p}$ is an epimorphism, hence there must be $\alpha(x)$ in $B(x)$ such that $\phi_{p}(\alpha(x))=\alpha_{p}(x)$. Hence $B(x) \subseteq(\alpha(x), p)$ and it is then immediate that $B(x)=(\alpha(x), p)$.

Conversely, suppose $B(x)=(\alpha(x), p)$ where $\phi_{p}(\alpha(x))$ is primitive in $R_{p}[x]$. Then, by Theorem $8, \phi_{p}(B(x))=R_{p}[x]$, hence $R[x]=B(x)$.

Case 2. $B(x)$ contains $\lambda$. Then, as in Case 1 , $R[x] / b(x) \simeq R_{\lambda}[x] / \phi_{\lambda}(b(x))$. Since $R_{\lambda}[x]$ is commutative, any ideal in $R_{\lambda}[x]$ which equals $R_{\lambda}[x]$ must be generated by an element which is congruent to 1 . The remainder of the proof now follows as in case 1 .

Theorem 9 is non-trivial. One example of an ideal equal to $R[x]$ is $(x+i, 3)$.

Theorem 9 indicates that the maximal ideals of $R[x]$ might not have the prime elements of $R[x]$ among their generators. This is indeed the case as will be shown in the following discussion which characterizes the maximal ideals of $R[x]$.

LEMMA 1. Let $g(x)$, not a unit, be in $Z[x]$. Then $(g(x))$ is not a maximal ideal in $R[x]$.

Proof. Since $Z[x]$ is noetherian it must contain a maximal ideal $(f(x), p)$, where $f(x)$ is irreducible $\bmod p$ and $p$ is prime in $z$, such that $(g(x))_{Z[x] \varsubsetneqq}(f(x), p)_{Z[x]}$. Let $\alpha(x)$ be any element in $(g(x))_{R[x]}$. Then, since $g(x)$ is in the center of $R[x]$, $\alpha(x)=g(x) \beta(x)$ for some $\beta(x)$ in $R[x]$. But $g(x)=f(x) g_{1}(x)+p h(x)$, where $g_{1}(x), h(x)$ are in $z[x]$. Hence $\alpha(x)=f(x) g_{1}(x) \beta(x)+p h(x) \beta(x)$ and $\alpha(x)$ is in $(f(x), p)_{R[x]}$. Thus $(g(x))_{R[x] \varsubsetneqq}(f(x), p)_{R[x]}$.

Case 1. $p \neq 2$. It suffices to show that $(f(x), p)_{R[x]} \neq R[x]$. Now the natural epimorphism $\phi_{p}$ will map $R[x] /(f(x), p)$ onto 
$R_{p}[x] /\left(\phi_{p}(f(x))\right)$. By Theorem 8 , since $f(x)$ is in $z[x],\left(\phi_{p}(f(x))\right.$ is a proper ideal in $R_{p}[x]$. Therefore $(f(x), p)$ must be a proper ideal in $R[x]$.

Case 2. $p=2$. Now $(f(x), 2)_{R[x]} \subseteq(f(x), \lambda)_{R[x]}$. Then, as in Case 1 , it follows that $(f(x), \lambda) \neq R[x]$.

LEMMA 2. Let $A(x)=\left(\alpha_{1}(x), \ldots, \alpha_{r}(x)\right)$ be a proper maximal ideal in $R[x]$. Then $A(x)$ contains a non-zero integer from $z$.

Proof. Let $a_{2}(x)=a_{0}^{(z)}(x)+a_{1}^{(z)}(x) i+a_{2}^{(z)}(x) j+a_{3}^{(z)}(x) k$, for $I \leq l \leq r$. Then, by the same argument that was used in Theorem 5 , $4 a_{0}^{(2)}(x), 4 a_{1}^{(2)}(x), 4 a_{2}^{(2)}(x), 4 a_{3}^{(2)}(x)$ are in $A(x)$ for $1 \leq 2 \leq r$. Thus $2 a_{0}^{(Z)}(x), 2 a_{1}^{(Z)}(x), 2 a_{2}^{(Z)}(x), 2 a_{3}^{(Z)}(x)$ are in $z[x]$, for $1 \leq l \leq r$, and their greatest common divisor in $z[x]$ must be 1 or 2 . Suppose not. Then there exists $g(x)$, not a unit, in $Z[x]$ such that $g(x)$ divides $a_{m}^{(l)}(x)$ for $0 \leq m \leq 3$ and $1 \leq l \leq 3$. Hence $g(x)$ divides $\alpha_{\eta}(x)$ for $1 \leq \imath \leq r$. But then $A(x) \subseteq(g(x)) \varsubsetneqq R[x]$. Since $A(x)$ is maximal it now follows that $A(x)=(g(x))_{R[x]}$, which is false by Lemma 1 .

Since the greatest common divisor in $Z[x]$ of the $2 a_{m}^{(Z)}(x)$ is 1 or 2 , there exists $t_{m}^{(2)}(x), 1 \leq \imath \leq r, 0 \leq m \leq 3$, in $Q[x]$ such that

$$
2 \sum_{l} \sum_{m} a_{m}^{(l)}(x) t_{m}^{(Z)}(x)=1 \text { or } 2 \text {. }
$$

Clearing denominators in the preceeding immediately gives the desired result.

LEMMA 3. Let $A(x)=\left(\alpha_{1}(x), \ldots, \alpha_{p}(x)\right)$ be a proper maximal ideal in $R[x]$. Then $A(x)$ contains either

(i) a prime integer $p \neq 2$ from 2 , or

(ii) an element from $R$ of norm two. 
Proof (i) (showing that $A(x)$ contains some prime integer $p$ ). By Lemma 2, $A(x)$ contains a non-zero integer $n$. Let the prime decomposition of $n$ in 2 be $p_{1} \cdots p_{m}$.

If $p_{1}$ is in $A(x)$ the proof is finished.

Suppose $p_{1}$ is not in $A(x)$. Since $A(x)$ is maximal it follows that $\left(A(x), p_{1}\right)=R[x]$. Hence there exists $\alpha(x)$ in $A(x)$ and $B(x)$ in $B(x)$ such that $\alpha(x)+B(x) p_{1}=1$. Thus

$$
\alpha(x) p_{2} \cdots p_{m}+\beta(x) n=p_{2} \ldots p_{m},
$$

so $p_{2} \cdots p_{m}$ is in $A(x)$. If $p_{2}$ is in $A(x)$, the proof is finished.

If not, by the same arguments as above, $p_{3} \ldots p_{m}$ is in $A(x)$.

Repeating the above argument, it must eventually follow that $p_{m}$ is in $A(x)$ if $p_{1}, \ldots, p_{m-1}$ are not.

(ii) If the prime integer obtained in (i) is odd the proof is finished.

Suppose the prime integer obtained in $(i)$ is 2 . Note that $2=\lambda \bar{\lambda}$. Suppose $\lambda$ is not in $A(x)$; then since $A(x)$ is maximal, $(A(x), \lambda)=R[x]$. Recalling that if $\lambda$ is a left divisor it is a right divisor and vice versa, there must exist $\alpha(x)$ in $A(x)$ and $\beta(x)$ in $R[x]$ such that $\alpha(x)+\beta(x) \lambda=1$. Thus $\alpha(x) \bar{\lambda}+\beta(x) 2=\bar{\lambda}$, so $\bar{\lambda}$ is in $A(x)$.

COROLLARY. Let $A(x)$ be a proper maximal ideal in $R[x]$. Then $A(x)$ must contain a proper maximal ideal from $R$.

Proof. This is immediate from Lemma 3.

Since all ideals in $R$ generated by elements of norm two are equal it follows from this corollary that $\lambda=1+i$ must be in $A(x)$.

LEMMA 4. Let $M(x)$ be a proper maximal ideal in $R[x]$. Then either

(i) $M(x)=(a(x), p)$, where $p$ is an odd prime in $z$ and $a(x) \neq 1 \bmod p$ is in $z[x]$ and irreducible $\bmod p$; or 
(ii) $M(x)=(\alpha(x), \lambda)$, where $N(\lambda)=2$ and $\alpha(x) \neq 1 \bmod \lambda$ is irreducible $\bmod \lambda$.

Proof. By Lemma $3, M(x)$ contains either a prime $p \neq 2$ or $\lambda=1+i$.

Case 1. $M(x)$ contains a prime $p \neq 2$. Let $M(x)=\left(p, \alpha_{1}(x), \ldots, \alpha_{p}(x)\right)$. Then since $(p)_{R[x]} \subseteq M(x)$, it follows that $R[x] / M(x) \cong R_{p}[x] / \phi_{p}(M(x))$, where $\phi_{p}$ is again the natural epimorphism from $R[x]$ to $R_{p}[x]$. Thus $\phi_{p}(M(x))$ is a proper ideal in $R_{p}[x]$. By Theorem 8, $\phi_{p}(M(x)) \subseteq\left(a_{p}(x)\right)$, for some $a_{p}(x)$ which is irreducible in $z_{p}[x]$. Hence $\phi_{p}\left(\alpha_{q}(x)\right)=a_{p}(x) \beta_{p}(x)$ for some $\beta_{p}(x)$ in $R_{p}[x]$, where $1 \leq l \leq r$. Therefore $\alpha_{q}(x)-a(x) \beta(x)$ must be in ${ }^{(p)} R[x], 1 \leq Z \leq r$, for some $\beta(x)$ in $R[x]$ and $a(x)$ irreducible in $z_{p}[x]$. Thus $\alpha_{q}(x)$ is in $(a(x), p)$ for $l \leq l \leq r$, and consequently $M(x) \subseteq(a(x), p) \subseteq R[x]$. But

$$
R[x] /(a(x), p) \cong R_{p}[x] /\left(\phi_{p}(a(x))\right)=R_{p}[x] /\left(a_{p}(x)\right),
$$

and $\left(a_{p}(x)\right) \neq R_{p}[x]$ so $(a(x), p) \neq R[x]$. Then, since $M(x)$ is maximal it must be that $M(x)=(a(x), p)$.

Case 2. $M(x)$ contains $\lambda$. Let $M(x)=\left(\lambda, \alpha_{1}(x), \ldots, \alpha_{p^{p}}(x)\right)$. Then, as in Case $1, \phi_{\lambda}(M(x)) \subseteq\left(\alpha_{\lambda}(x)\right)$ for some $\alpha_{\lambda}(x)$ irreducible in $R_{\lambda}[x]$. Thus, since $R_{\lambda}[x]$ is commutative, for some $\beta_{\lambda}(x)$ in $R_{\lambda}[x]$, $\dot{\phi}_{\lambda}\left(\alpha_{\eta}(x)\right)=\alpha_{\eta}(x) \beta_{\lambda}(x)$ where $I \leq \ell \leq r$. The argument is now completed in a similar fashion to Case 1 .

LEMMA 5. (i) Let $p$ be an odd prime and $a(x) \neq 1 \bmod p$ be in $Z[x]$ and irreducible $\bmod p$. Then $M(x)=(a(x), p)$ is a proper maximal ideal in $R[x]$.

(ii) Let $\lambda=1+i$ and $\alpha(x)$ | 1 mod $\lambda$ in $R[x]$ be irreducible mod $\lambda$. Then $M(x)=(\alpha(x), \lambda)$ is a proper maximal ideal in $R[x]$.

Proof. (i) Suppose $(a(x), p)$ is not a maximal ideal in $R[x]$. Since $R[x]$ is noetherian there must exist a maximal ideal $N_{1}(x)$ in 
$R[x]$ such that $(p, \alpha(x)) \varsubsetneqq N_{1}(x) \varsubsetneqq R[x]$. By Lemma $3, N_{1}(x)$ must contain either an odd prime or $\lambda$. Since $N_{1}(x) \neq R[x]$ it is clear that $N_{1}(x)$ can not contain $\lambda$ or any odd prime except $p$. Thus, by Lemma 4 , $N_{1}(x)=(b(x), p)$, where $b(x)$, not a unit, is in $Z[x]$ and irreducible $\bmod p$.

Since $a(x)$ is in $(b(x), p)=N_{1}(x)$, there must exist $\alpha(x)$ and $\beta(x)$ in $R[x]$ such that $a(x)=b(x) \beta(x)+p \alpha(x)$. Hence $\phi_{p}(a(x))=\phi_{p}\left(b(x) \phi_{p}(\beta(x))\right)$ in $R_{p}[x]$. But $a(x)$ is irreducible mod $p$, hence $\phi_{p}(\alpha(x))$ must be irreducible in $R_{p}[x]$; thus $\phi_{p}(\beta(x))$ must be a unit in $R_{p}[x]$. Let $\gamma_{p}(x)$ be its inverse in $R_{p}[x]$; then since $\phi_{p}$ is an epimorphism there must be a $\gamma(x)$ in $R[x]$ such that $\phi_{p}(\gamma(x))=\gamma_{p}(x)$. Hence $\gamma(x) a(x)-b(x)$ is in $(p)$ in $R[x]$. Thus $b(x)$ is in $(a(x), p)$. But then $(a(x), p)=N_{1}(x)$, which is a contradiction.

(ii) Suppose $(a(x), \lambda)$ is not a maximal ideal in $R[x]$. Then it must be contained in a maximal ideal $N_{1}(x)$. By Lemma $3, N_{1}(x)$ must contain either an odd prime from $Z$ or $\lambda$. Since $N_{1}(x) \neq R[x]$ it is clear that $N_{1}(x)$ can not contain an odd prime $p$. Thus $N_{1}(x)$ must be of the form $(\beta(x), \lambda)$ where $\beta(x) \neq 1 \bmod \lambda$ and $\beta(x)$ is irreducible $\bmod \lambda$. Hence $(\alpha(x), \lambda) \subseteq(\beta(x), \lambda)$; so $\left(\phi_{\lambda}(\alpha(x))\right) \subseteq\left(\phi_{\lambda}(\beta(x))\right)$ in $R_{p}[x]$. But $\alpha(x)$ is irreducible $\bmod \lambda$, so $\left(\phi_{\lambda}(\alpha(x))\right)$ is a maximal ideal in $R_{\lambda}[x]$; hence $\left(\phi_{\lambda}(\alpha(x))\right)=\left(\phi_{\lambda}(\beta(x))\right)$. Returning to $R[x]$ it follows that $(\alpha(x), \lambda)=(\beta(x), \lambda)=N_{1}(x)$, which is a contradiction.

THEOREM 10. $M(x)$ is a proper maximal ideal in $R[x]$, if, and only if, either

(i) $M(x)=(a(x), p)$, where $p$ is an odd prime in $z$ and $a(x) \neq 1 \bmod p$ in $z[x]$ is irreducible $\bmod p ;$ or

(ii) $M(x)=(\alpha(x), \lambda)$, where $N(\lambda)=2$ and $\alpha(x)$ f $1 \bmod \lambda$ is irreducible $\bmod \lambda$. 
Proof. Immediate by Lemmas 4 and 5 .

The preceding discussion showed that the maximal ideals were not, as might be expected, generated by the prime elements of $R[x]$. The following discussion will show that the unexpected also happens in the characterization of the prime ideals. Again, as for the maximal ideals, a characterization surprisingly analogous to the situation in $z[x]$ will be shown to occur.

LEMMA 6. Let $P(x)$ be a prime ideal in $R[x]$. Then $P(x) \cap R$ is a prime ideal in $R$.

Proof. Suppose $P(x) \cap R$ is not a prime ideal in $R$. Then there exist ideals $A$ and $B$ in $R$ such that $A B \subseteq P(x) \cap R$, but neither $A$ nor $B$ is in this intersection. Now raise the ideals $A$ and $B$ to $R[x]$ forming the ideals $A(x)$ and $B(x)$. Then $A(x)=(\alpha)$ and $B(x)=(\beta)$ for some $\alpha$ and $\beta$ in $R$.

Let $\gamma(x)$ be any element in $A(x) B(x)$. Then

$$
\gamma(x)=\left[\sum_{l=1}^{n} \gamma_{l}^{(1)}(x) \alpha \gamma_{l}^{(2)}(x)\right]\left[\sum_{h=1}^{m} \gamma_{h}^{(3)}(x) B \gamma_{h}^{(4)}(x)\right],
$$

where $\gamma_{l}^{(1)}(x), \gamma_{l}^{(2)}(x), \gamma_{h}^{(3)}(x), \gamma_{h}^{(4)}(x), 1 \leq l \leq n, I \leq h \leq m$, are in $R[x]$. Thus $\gamma(x)$ is a polynomial with coefficients in $A B$. Hence $A(x) B(x) \subseteq P(x)$, which is prime. Without loss of generality, suppose $A(x) \subseteq P(x)$; then $A \subseteq A(x) \cap R \subseteq P(x) \cap R$, which is a contradiction.

LEMMA 7. Let $m$ be in $Z, a(x)$ be in $Z[x]$, and $\alpha(x), \beta(x)$ be in $R[x]$. If $\operatorname{ma}(x)=\alpha(x) \beta(x)$ and $\alpha(x)$ is irreducible in $Z[x]$, then $m$ divides $\beta(x)$.

$$
\text { Proof. Let } a(x)=a_{0}+a_{1} x+\ldots+a_{n_{1}}^{x_{1}} \text { in } z[x] \text {, }
$$
$\beta(x)=\beta_{0}+\ldots+\beta_{n_{2}} x^{n_{2}}$ in $R[x]$ and $p_{1} \cdots p_{q}$ be the prime factorization of $m$ in $Z$. Since $a(x)$ is irreducible in $Z[x]$, there must exist a first coefficient, say $a_{s}$, such that $p_{1}$ does not divide $a_{s}$ in $Z$. 
Suppose $p_{1}$ does not divide $B(x)$ in $R[x]$. Then there exists a first coefficient, say $\beta_{t}$, such that $p_{1}$ does not divide $\beta_{t}$ in $R$. Now the-coefficient of $x^{s+t}$ in $a(x) B(x)$ is

$$
a_{0} \beta_{s+t}+a_{1} \beta_{s+t-1}+\ldots+a_{s} \beta_{t}+\ldots+a_{s+t} \beta_{0} .
$$

Since this coefficient is divisible by $p_{1}$ and $a_{0}, \ldots, a_{s-1}$, $\beta_{t-1}, \ldots, \beta_{0}$ are divisible by $p_{1}$ it follows that $p_{1}$ divides $a_{s} \beta_{t}$ in $R$. But since $p_{1}$ is prime and $p_{1}$ does not divide $a_{s}$, there exist $c_{1}$ and $c_{2}$ in $Z$ such that $c_{1} p_{1}+c_{2} a_{s}=1$. Hence $c_{1} p_{1} \beta_{t}+c_{2} a_{s} \beta_{t}=\beta_{t}$, so that $p$ divides $B_{t}$ in $R$, which is a contradiction. Hence $p_{1}$ divides $\beta(x)$ in $R[x]$.

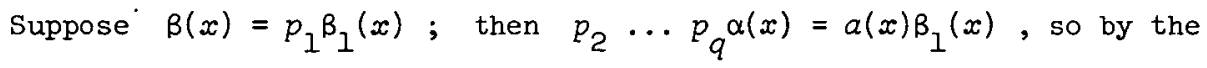
same argument as above $p_{2}$ must divide $\beta_{1}(x)$. Continuing in this fashion it follows that $m$ divides $\beta(x)$.

COROLLARY. Let $p$ be prime in $Z, a(x)$ be in $Z[x]$ and $\alpha(x)$, $\beta(x)$ be in $R[x]$. If $p \alpha(x)=\alpha(x) \beta(x)$ in $R[x]$ and $p$ does not divide $a(x)$, then $p$ divides $\beta(x)$.

Proof. Let $a(x)=a_{0}+a_{1} x+\ldots+a_{n} x^{n}$ in $2[x]$ and $\beta(x)=\beta_{0}+\ldots+\beta_{m} x^{m}$ in $R[x]$. Since $p$ does not divide $a(x)$ there must exist a first coefficient, say $a_{s}$, such that $p$ does not divide $a_{s}$ in $Z$.

Now suppose $p$ does not divide $\beta(x)$ in $R[x]$ and obtain a contradiction as in Lemma 7 .

LEMMA 8. Let $P(x)$ be a proper prime ideal in $R[x]$. Then $P(x)$ must have one of the following forms:

(i) $(p(x))$, where $p(x)$ is irreducible in $Z[x]$;

(ii) (P), where $P$ is a prime ideal in $R$;

(iii) $(a(x), p)$, where $p$ is an odd prime in $z$ and $a(x) \neq 1 \bmod p$ in $Z[x]$ is irreducible $\bmod p$; 
(iv) $(a(x), \lambda)$, where $N(\lambda)=2$ and $\alpha(x) \neq 1 \bmod \lambda$ is irreducible mod $\lambda$.

Proof. By Lemma 6, $P(x) \cap R$ is a prime ideal in $R$. Thus, by Theorem 2, there are three cases to consider.

Case 1. $P(x) \cap R=\{0\}$. First raise $P(x)$ to be an ideal in $K[x]$. Since $P(x) \cap R=\{0\}$ this must be a proper ideal in $K[x]$; so, by Theorem 5, $P(x)_{K[x]}=(a(x))$ for some $a(x)$ in $Z[x]$. Hence $a(x)$ can be written as a $K[x]$ linear combination of generators for $P(x)$. But then there exists a $d$ in $Z$ such that $d a(x)$ can be written as an $R[x]$ linear combination of generators for $P(x)$, so that $d a(x)$ is in $P(x)$. Since $d$ and $a(x)$ are in the center of $R[x]$ it follows that the ideal product $(d)(a(x))$ is in $P(x)$. But $P(x)$ is prime and $P(x) \cap R=\{0\}$; therefore $(a(x)) \subseteq P(x)$.

Let $a_{1}(x) \ldots a_{n}(x)$ be the prime factorization of $a(x)$ in $Z[x]$. Then one of the ideals $\left(a_{\eta}(x)\right), 1 \leq \ell \leq n$, must be in $P(x)$. Without loss of generality, suppose $\left(a_{1}(x)\right) \subseteq P(x)$. Then it remains to show that $P(x) \subseteq\left(a_{1}(x)\right)$. Suppose the generators of $P(x)$ are $\alpha_{1}(x), \ldots, \alpha_{r}(x)$. Since $P(x)_{K[x]}=(a(x))=\left(a_{1}(x) \ldots a_{n}(x)\right)_{K[x]}$ it follows that there exist integers $m_{1}, \ldots, m_{r}$ in $Z$ such that $m_{h} \alpha_{h}(x)=a_{1}(x) \beta_{h}(x)$, $l \leq h \leq r$, where $B_{h}(x)$ is in $R[x]$ and $a_{1}(x)$ is irreducible in $z[x]$. By Lemma $7, m_{\eta}$ divides $B_{h}(x)$ in $R[x]$ for $1 \leq h \leq r$. Thus $\alpha_{h}(x), 1 \leq h \leq r$, is in the ideal $\left(a_{1}(x)\right)$ in $R[x]$; so $P(x) \subseteq\left(a_{1}(x)\right)$.

Hence $P(x)=\left(a_{1}(x)\right)$, where $a_{1}(x)$ is irreducible in $Z[x]$.

Case 2. $P(x) \cap R=P$, where $P \neq\{0\}$ is a proper prime ideal in $R$.

(i) $P=(p)$ where $p$ is an odd prime in $Z$. The first step is to show that $\phi_{p}(P(x))$ is a prime ideal in $R_{p}[x]$. Let $\left(a_{p}(x)\right)$ and $\left(b_{p}(x)\right)$ be proper ideals in $R_{p}[x]$ such that $\left(a_{p}(x)\right)\left(b_{p}(x)\right) \subseteq \phi_{p}(P(x))$. Then $a_{p}(x) b_{p}(x)$ is in $\phi_{p}(P(x))$, so that $a_{p}(x) b_{p}(x)+\alpha(x) p$ is in 
$P(x)$ for some $\alpha(x)$ in $R[x]$. But $p$ is in $P(x)$, so $a_{p}(x) b_{p}(x)$ must be in $P(x)$. Since $a_{p}(x)$ and $b_{p}(x)$ are both in the center of $R[x]$ and $P(x)$ is prime it must be that $a_{p}(x)$ or $b_{p}(x)$ is in $P(x)$. Hence $\left(a_{p}(x)\right)$ or $\left(b_{p}(x)\right)$ must be in $\phi_{p}(P(x))$ and thus $\phi_{p}(P(x))$ is a prime ideal in $R_{p}[x]$.

By the above the prime ideals in $R[x]$ containing $p$ must lie among the inverse images with respect to $\phi_{p}$ of the prime ideals in $R_{p}[x]$. But the only ideals in $R[x]$ which contain $p$ and are among these inverse images are $(p)$ and $(\alpha(x), p)$, where $a(x)$ is in $Z[x]$ and irreducible $\bmod p$.

(ii) $P=(\lambda)$ where $N(\lambda)=2$. Then, since $\lambda$ is in $P(x)$, the isomorphism $R[x] / P(x) \cong R_{\lambda}[x] / \phi_{\lambda}(P(x))$ holds. But $R_{\lambda}[x]$ is a commutative ring; thus $P(x)$ is a prime ideal in $R[x]$, if, and only if, $\phi_{\lambda}(P(x))$ is a prime ideal in $R_{p}[x]$. Thus the prime ideals in $R[x]$ containing $\lambda$ must be among the inverse images with respect to $\phi_{\lambda}$ of the prime ideals in $R_{\lambda}[x]$. Consequently, the only possibilities are $(\lambda)$ and $(\alpha(x), \lambda)$, where $\alpha(x)$ in $R[x]$ is irreducible mod $\lambda$.

Case 3. $P(x) \cap R=R$. If this is true, then 1 is in $P(x)$ which is impossible.

LEMMA 9. (i) Let $p$ be an odd prime in $z$ and $a(x)$ f 1 mod $p$ in $z[x]$ be irreducible $\bmod p$. Then $(a(x), p)$ is a proper prime ideal in $R[x]$.

(ii) Let $N(\lambda)=2$ and $\alpha(x)$ in $R[x]$ be irreducible $\bmod \lambda$. Then $(\alpha(x), \lambda)$ is a proper prime ideal in $R[x]$.

Proof. (i) Let $C(x)$ and $B(x)$ be two ideals in $R[x]$ such that $C(x) B(x) \subseteq(a(x), p)$. Then

$$
\phi_{p}(C(x)) \phi_{p}(B(x)) \subseteq \phi_{p}(a(x), p)=\phi_{p}(a(x))=A_{p}(x)
$$

say. By Theorem 8, $A_{p}(x)$ is a prime ideal in $R_{p}[x]$. Without loss of generality $\phi_{p}(B(x)) \subseteq A_{p}(x)$. Then 


$$
B(x) \subseteq \phi_{p}^{-1} \phi_{p}(B(x)) \subseteq \phi_{p}^{-1}\left(A_{p}(x)\right) \subseteq(a(x), p)
$$

for, by Theorem 10, $(a(x), p)$ is a maximal ideal. Thus $(a(x), p)$ is a prime ideal.

(ii) Follows by the same argument as was used in (i).

LEMMA 10. (i) Let $p$ be an odd prime in $Z$. Then $(p)$ is a proper prime ideal in $R[x]$.

(ii) Let $N(\lambda)=2$. Then ( $\lambda$ ) is a proper prime ideal in $R[x]$.

Proof. (i) Let $A(x)$ and $B(x)$ be two ideals in $R[x]$ such that $A(x) B(x) \subseteq(p)$. Then, in $R_{p}[x], \phi_{p}(A(x)) \phi_{p}(B(x)) \subseteq(0)$.

Case 1. At least one of $\phi_{p}(A(x))$ or $\phi_{p}(B(x))$ is (0). Without loss of generality suppose it is $\phi_{p}(A(x))$. Then $A(x) \subseteq \phi_{p}^{-1}\left(\phi_{p}(A(x))\right) \subseteq(p)$ and the proof is complete.

Case 2. $\phi_{p}(A(x))$ and $\phi_{p}(B(x))$ are both proper ideals in $R_{p}[x]$. By Theorem 8, there exist $a_{p}(x)$ and $b_{p}(x)$ in $z_{p}[x]$ such that $\phi_{p}(A(x))=\left(a_{p}(x)\right)$ and $\phi_{p}(B(x))=\left(b_{p}(x)\right)$. Then, since $\left(a_{p}(x)\right)\left(b_{p}(x)\right) \subseteq(0), p$ must divide $a_{p}(x) b_{p}(x)$ in $Z[x]$. Consequently, without loss of generality, $p$ divides $a_{p}(x)$ in $Z[x]$. Thus $\left(a_{p}(x)\right)=(0)$; so $A(x) \subseteq \phi_{p}^{-1}\left(\phi_{p}(A(x))\right) \subseteq(p)$ and the proof is complete.

Case 3. Either $\phi_{p}(A(x))$ or $\phi_{p}(B(x))$ is $R_{p}[x]$. Without loss of generality, suppose $\phi_{p}(A(x))=R_{p}[x]$. Then, by Theorem 8 , it must be generated by a primitive element in $R_{p}[x]$. Thus the generator of $\phi_{p}(B(x))$ must be divisible by $p$; so $\phi_{p}(B(x))=(0)$, and again the proof is complete.

(ii) Let $A(x)$ and $B(x)$ be two ideals in $R[x]$ such that $A(x) B(x) \subseteq(\lambda)$. Then $\phi_{\lambda}(A(x)) \phi_{\lambda}(B(x)) \subseteq(0)$ in $R_{\lambda}[x]$. Since $R_{\lambda}[x]$ is a commutative integral domain it follows, without loss of generality, 
that $\phi_{\lambda}(A(x)) \subseteq(0)$. Thus $A(x) \subseteq \phi_{\lambda}^{-1} \phi_{\lambda}(A(x)) \subseteq(\lambda)$, and the proof is complete.

LEMMA 11. Let $p(x)$, not equal to a constant, be irreducible in $Z[x]$. Then $(p(x))$ is a prime ideal in $R[x]$.

Proof. Let $A(x)$ and $B(x)$ be ideals in $R[x]$ such that $A(x) B(x) \subseteq(p(x))$. Then, lifting each of these ideals to $K[x]$, it

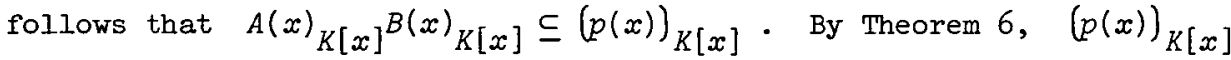
is a prime ideal in $K[x]$. Without loss of generality, suppose $A(x)_{K[x]} \subseteq(p(x))_{K[x]}$.

Let $\alpha_{1}(x), \ldots, \alpha_{p}(x)$ be the generators of $A(x)$ in $R[x]$. Then $\alpha_{Z}(x)=p(x) \rho_{\eta}(x), \quad 1 \leq Z \leq r, \rho_{\eta}(x)$ in $K[x]$; so $m_{l} \alpha_{l}(x)=p(x) \beta_{l}(x), 1 \leq l \leq r, \beta_{l}(x)$ in $R[x]$, and $m_{l}$ in $Z$. Hence, by Lemma $7, m_{l}$ divides $\beta_{\eta}(x)$ in $R[x]$ for $1 \leq \ell \leq r$. Thus $\alpha_{2}(x)$ is in $(p(x))$ for $l \leq l \leq r$. Hence $A(x) \subseteq(p(x))$ and $(p(x))$ is a prime ideal in $R[x]$.

THEOREM 11. $P(x)$ is a proper prime ideal in $R[x]$, if, and only if, one of the following is true:

(i) $P(x)=(p(x))$, where $p(x)$, not a unit, is irreducible in $Z[x]$;

(ii) $P(x)=(P)$, where $P$ is a proper prime ideal in $R$;

(iii) $P(x)=(a(x), p)$, where $p$ is an odd prime in $Z$ and $a(x) \neq 1 \bmod p$ in $z[x]$ is irreducible $\bmod p$;

(iv) $P(x)=(\alpha(x), \lambda)$, where $N(\lambda)=2$ and $\alpha(x) \neq 1 \bmod \lambda$ is in $R[x]$ and irreducible $\bmod \lambda$.

Proof. This is immediate from Lemmas 8 through 11.

\section{A Szekeres type basis for the ideals of $R[x]$}

DEFINITION. Let $A(x)$ be an ideal in $R[x] \cdot A(x)$ is a primitive ideal if there does not exist an ideal $(a(x))$, where $a(x)$ is in $Z[x]$ or $N(a(x))=2$, such that $A(x) \subseteq(a(x)) \varsubsetneqq R[x]$.

Let $\alpha(x)$ be an element in $R[x]$. Then 


$$
2 \alpha(x)=a_{0}(x)+a_{1}(x) i+a_{2}(x) j+a_{3}(x) k
$$

for some $a_{0}(x), a_{1}(x), a_{2}(x), a_{3}(x)$ in $Z[x]$. Let $a(x)$ be the greatest common divisor of $a_{0}(x), \ldots, a_{3}(x)$ in $Z[x]$. Then

$$
2 \alpha(x)=a(x)\left(b_{0}(x)+b_{1}(x) i+b_{2}(x) j+b_{3}(x) k\right)=a(x) \beta(x),
$$

where $B(x)$ is in $R[x]$, its coordinates are in $Z[x]$, and have no common divisor there. Then there are two possibilities:

(i) two divides $a(x)$ in $Z[x]$; then, clearly, $\frac{a(x)}{2}$ is the largest element in $Z[x]$ which divides $\alpha(x)$ in $R[x]$;

(ii) two does not divide $a(x)$ in $Z[x]$; then, by the corollary to Lemma 7 , two must divide $\beta(x)$ in $R[x]$. Hence, $a(x)$ is the largest element in $Z[x]$ which divides $\alpha(x)$ in $R[x]$.

Now let $B(x)=\left(\beta_{1}(x), \ldots, \beta_{s}(x)\right)$ be any ideal in $R[x]$. By the preceding paragraph, for each $\beta(x), I \leq l \leq s$, there is a greatest $a_{\eta}(x)$ in $z[x]$ such that $\beta_{\eta}(x)=a_{\eta}(x) \gamma_{\eta}(x), \gamma_{\eta}(x)$ in $R[x]$. Now let $a(x)$ be the greatest common divisor of the $a_{\eta}(x), 1 \leq l \leq s$, in $z[x]$. Then

$$
B(x)=(a(x))\left(\Upsilon_{1}(x), \ldots, \Upsilon_{s}(x)\right)
$$

Let $\gamma_{l}(x)=\gamma_{0}^{(l)}+\gamma_{l}^{(l)} x+\ldots+\gamma_{m_{l}}^{(l)} x^{m_{l}}, I \leq l \leq s$. Factor from the $\gamma_{h}^{(l)}, \quad l \leq l \leq s, 0 \leq h \leq m_{l}$, all common factors $\lambda$ in $R$ with norm two. Let $\gamma_{2}(x)=\lambda_{1} \ldots \lambda_{t} \alpha_{l}(x), 1 \leq \tau \leq s$, and $N\left(\lambda_{1}\right)=\ldots=N\left(\lambda_{t}\right)=2$. Then

$$
B(x)=(a(x))\left(\lambda_{1}\right) \ldots\left(\lambda_{t}\right)\left(\alpha_{1}(x), \ldots, \alpha_{s}(x)\right)=(a(x))(\lambda)^{t} A(x),
$$

where $t$ is a non-negative integer and $A(x)=\left(\alpha_{1}(x), \ldots, \alpha_{s}(x)\right)$. Then $A(x)$ is a primitive ideal in $R[x]$.

Thus, in order to characterize all the ideals in the ring $R[x]$, it 
is sufficient to characterize the primitive ideals. This will be done by adapting a proof by Szekeres [3].

LEMMA 12. Let $A(x)$ be a primitive ideal in $R[x]$. Then $A(x)$ contains a non zero integer from $Z$.

Proof. Let $A(x)=\left(\alpha_{1}(x), \ldots, \alpha_{p}(x)\right)$ where

$$
\alpha_{\eta}(x)=a_{0}^{(Z)}(x)+a_{1}^{(l)}(x) i+a_{2}^{(l)}(x) j+a_{3}^{(Z)}(x) k
$$

for $1 \leq z \leq r$. Then, by the same argument as in Theorem $5,4 a_{m}^{(z)}(x)$, $1 \leq Z \leq r, 0 \leq m \leq 3$, are in $A(x) \cap Z[x]$. Moreover, since $A(x)$ is primitive, the greatest common divisor in $Z[x]$ of these elements must be 2 or 4 . Thus, there exist $h_{m}^{(l)}(x), 1 \leq Z \leq r, 0 \leq m \leq 3$, in $Q[x]$ such that $4 \sum_{l} \sum_{m} a_{m}^{(Z)}(x) h_{m}^{(Z)}(x)$ is 2 or 4 . Clearing denominators, it follows that

$$
\sum_{i} \sum_{m} 4 a_{m}^{(2)}(x) k_{m}^{(2)}(x)=k \neq 0
$$

in $Z$, where the $k_{m}^{(Z)}(x), I \leq Z \leq r, 0 \leq m \leq 3$, are in $Z[x]$. Hence $k$ is in $A(x)$.

DEFINITION. Let $\alpha$ and $\beta$ be in $R$. Then $\alpha$ is equivalent to $\beta(\alpha \sim \beta)$, if, and only if, $(\alpha)=(\beta)$.

In each equivalence class of $R$ defined above choose a certain element. This will be called a normed element of $R$.

Now the oniy proper ideals in $R$ are of the form $\left(m \lambda^{t}\right)$ where $m$ is non negative in $Z, N(\lambda)=2$, and $t=0$ or 1 . Thus one complete representative set of the normed $R$ is

$$
N=\{0,1,2, \ldots, \lambda, 2 \lambda, 3 \lambda, \ldots\} \text {. }
$$

For convenience let $\bar{N}=\{0,1,2, \ldots, \bar{\lambda}, 2 \bar{\lambda}, 3 \bar{\lambda}, \ldots\}$.

LEMMA 13. Let $A \subseteq B$ be ideals in $R$ and $A=\left(\gamma_{1}\right)$, where $\gamma_{1}$ is given in $N \cup \bar{N}$. Then there exists a $\gamma_{2}$ in $N \cup \bar{N}$ such that $B=\left(\gamma_{2}\right)$ 
and $\gamma_{1}=\alpha \gamma_{2}$ where $\alpha$ is in $N$.

Proof. Clearly $\gamma_{2}$ can be chosen in $N \cup \bar{N}$ so that $B=\left(\gamma_{2}\right)$ and $\gamma_{2}$ can be either of the form $m_{2} \lambda^{t_{2}}$ or $m_{2} \bar{\lambda}^{t^{2}}$. It just remains to show that given $\gamma_{1}$ and the fact that $\gamma_{1}=\alpha_{1} m_{2} \lambda^{t_{2}}=\alpha_{2} m_{2} \bar{\gamma}^{t_{2}}$, at least one of the $\alpha_{1}$ or $\alpha_{2}$ is in $N$.

Case 1. $\gamma_{1}$ is in $N$. Let $\gamma_{1}=m_{1} \gamma_{1}^{t_{1}}=\alpha_{1} m_{2} \lambda^{t_{2}}=\alpha_{2} m_{2} \bar{\lambda}^{t_{2}}$.

(i) $t_{1}=t_{2}=0$. Then $m_{1}=\alpha_{1} m_{2}$, so $m_{1}^{2}=N\left(\alpha_{1}\right) m_{2}^{2}$ in 2 and $m_{2}$ must divide $m_{1}$. Thus $\alpha_{1}$ is in $N$ for $\gamma_{2}=m_{2}$.

(ii) $t_{1}=0, t_{2}=1$. Then $m_{1}=\alpha_{2} m_{2} \bar{\lambda}$, so $m_{1}^{2}=2 N\left(\alpha_{2}\right) m_{2}^{2}$ in $A$. Thus $m_{1}=k m_{2}$ for some $k$ in $z$; hence $k^{2}=N\left(\alpha_{2}\right)_{2}$, so $k$ must be even. Let $k=2 k_{1}$. Then $2 k_{1}=\alpha_{2} \bar{\lambda}$, so $k_{1} \lambda=\alpha_{2}$; that is, $\alpha_{2}$ is in $N$ if $\gamma_{2}=m_{2} \bar{\lambda}$.

(iii) $t_{1}=1, t_{2}=0$. Then $m_{1} \lambda=\alpha_{1} m_{2} ;$ so $2 m_{2}^{2}=N\left(\alpha_{1}\right) m_{2}^{2}$ in $A$. Hence $m_{2}$ divides $m_{1}$ in $Z$. Thus $\alpha_{1}$ is in $N$ if $\gamma_{2}=m_{2}$.

(iv) $t_{1}=t_{2}=1$. Then $m_{1} \lambda=\alpha_{1} m_{2} \lambda$; so $m_{1}=\alpha_{1} m_{2}$ and the proof is as in (i).

Case 2. $\gamma_{1}$ is in $\bar{N}$. Let $\gamma_{1}=m_{1} \bar{\lambda}_{1}^{t_{1}}$. Then the same type of argument that was used in Case I holds.

Let $R(\alpha)$ be the system of representatives containing the element 0 , of the residue classes $\bmod \alpha$, for an element $\alpha$ in $N$.

THEOREM 12. Let $A(x)$ be a primitive ideal in $R[x]$. Then $A(x)=\left(\alpha_{0}(x), \ldots, \alpha_{m}(x)\right)$, where

(i) $\alpha_{0}(x)=\alpha_{1} \ldots \alpha_{m}$, 


$$
\alpha_{\eta} \alpha_{\eta}(x)=x \alpha_{\eta-1}+\sum_{h=1}^{\eta} \beta_{h l_{h-1}}(x), \quad 1 \leq \eta \leq m ;
$$

(ii) $\alpha_{1}, \ldots, \alpha_{m}$ are in $N, \alpha_{2} \neq 0$, and $\alpha_{m} \neq 1$;

(iii) $\beta_{12}, \ldots, \beta_{2 l}$ are in $R\left(\alpha_{2}\right)$ for $1 \leq 2 \leq m$.

Proof I (showing that $\alpha_{0}(x), \ldots, \alpha_{m}(x)$ are in $R[x]$ ). Obviously $\alpha_{0}(x)$ is in $R[x]$.

$$
\begin{aligned}
\alpha_{1} \alpha_{1}(x) & =x \alpha_{0}(x)+\beta_{11} \alpha_{0}(x) \\
& =\left(x+\beta_{11}\right) \alpha_{1} \ldots \alpha_{m} \\
& =\alpha_{1}\left(x+\beta_{11}^{\prime}\right) \alpha_{2} \ldots \alpha_{m},
\end{aligned}
$$

where $\beta_{11}^{\prime}$ is in $R$. Thus $\alpha_{1}(x)=\left(x+\beta_{11}^{\prime}\right) \alpha_{2} \ldots \alpha_{m}$ and is in $R[x]$. Moreover $\alpha_{1}(x)$ has leading coefficient $\alpha_{2} \ldots \alpha_{m}$.

$$
\text { (ii) } \begin{aligned}
\alpha_{2} \alpha_{2}(x) & =x \alpha_{1}(x)+\beta_{12} \alpha_{0}(x)+\beta_{22} \alpha_{1}(x) \\
& =\left(x+\beta_{22}\right)\left(x+\beta_{11}^{\prime}\right) \alpha_{2} \ldots \alpha_{m}+\beta_{12} \alpha_{1} \ldots \alpha_{m} \\
& =\alpha_{2}\left(x+\beta_{22}^{\prime}\right)\left(x+\beta_{11}^{\prime \prime}\right) \alpha_{3} \ldots \alpha_{m}+\alpha_{2} \beta_{12}^{\prime} \alpha_{1}^{\prime} \alpha_{3} \ldots \alpha_{m},
\end{aligned}
$$

where $\beta_{22}^{\prime}, \beta_{11}^{\prime \prime}, \beta_{12}^{\prime}, \alpha_{1}^{\prime}$ are in $R$. Thus $\alpha_{2}(x)$ is in $R[x]$ and has leading coefficient $\alpha_{3} \ldots \alpha_{m}$.

(iii) Continuing in this fashion it follows that $\alpha_{0}(x), \ldots, \alpha_{m}(x)$ are in $R[x]$. The leading coefficient of $\alpha_{\eta}(x), 1 \leq l \leq m$, is $\alpha_{\eta+1} \cdots \alpha_{m}$ and the leading coefficient of $\alpha_{m}(x)$ is 1 .

II (showing that $\left(\alpha_{0}(x), \ldots, \alpha_{m}(x)\right)$ is indeed a primitive ideal). Since $\alpha_{m}(x)$ has leading coefficient 1 and $\alpha_{0}(x)$ is a constant other than zero it is obvious that for $m>0$, the ideal $\left(\alpha_{0}(x), \ldots, \alpha_{m}(x)\right)$ is primitive. For $m=0$, the polynomial sequence $\alpha_{0}(x), \ldots, \alpha_{m}(x)$ is reduced to $\alpha_{0}(x)=I$; so $\left(\alpha_{0}(x), \ldots, \alpha_{m}(x)\right)$ is again primitive.

III. Let $M_{Z}(x)$ be the two-sided $R$-module consisting of those elements of $A(x)$ whose degree is at most $\tau$. Then 


$$
M_{0}(x) \subseteq M_{1}(x) \subseteq M_{2}(x) \subseteq \cdots
$$

Furthermore, the leading coefficients of the elements of $M_{Z}(x)$ form an ideal $M_{2}=\left(\gamma_{2}\right)$ in $R$. By Lemma 12, $M_{0} \neq\{0\}$; thus

$$
M_{0} \subseteq M_{1} \subseteq M_{2} \subseteq \cdots
$$

is a non-trivial chain.

IV. Since $R[x]$ is a noetherian ring, $A(x)$ is finitely generated. Consequently, there is a minimal $l$ for which $A(x)$ is generated by the elements of $M_{\eta}(x)$. Denote this $\tau$ by $m(A(x))=m$.

V. Now choose, in one way or another, from among each of the $M_{0}(x), \ldots, M_{m}(x)$ a polynomial $\alpha_{z}(x)=\gamma_{2} x^{2}+\ldots, 0 \leq z \leq m$. Then, for each element $\alpha(x)$ of $M_{\ell}(x), \quad>0$, since its leading coefficient is in $M_{Z}$ which is principal, there is an $\alpha$ in $R$ for which $\alpha(x)=\alpha \alpha_{\eta}(x)$ lies in $M_{l-1}(x)$. Then, since the degrees of $\alpha_{\eta}(x), \ldots, \alpha_{0}(x)$ are descending, it follows by induction that $\alpha_{0}(x), \ldots, \alpha_{q}(x)$ constitute a left $R$-basis of the $R$-module $M_{\eta}(x)$. Moreover, by definition of $m$,

$$
A(x)=\left(\alpha_{0}(x), \cdots, \alpha_{m}(x)\right) .
$$

VI. By III, $M_{0} \subseteq M_{1} \subseteq M_{2} \subseteq \ldots$. Each of these ideals is principal in $R$ and the generator $\gamma_{0}$ of $M_{0}$ can be taken in $N$. Then, by Lemma 13, there exists $\gamma_{1}$ in $N \cup \bar{N}$ such that $M_{1}=\left(\gamma_{1}\right)$ and $\gamma_{0}=\alpha_{1} \gamma_{1}$ where $a_{1}$ is in $N$. Continuing up this ideal chain applying Lemma 13, it follows that there exist elements $\alpha_{1}, \ldots, \alpha_{m} \neq 0$ in $N$ such that

$$
\alpha_{\eta-1}=\alpha_{\eta} \gamma_{\eta}, \quad l \leq Z \leq m \text {. }
$$

VII. By VI, $\alpha_{\eta} \gamma=\gamma_{Z-1}$ for $1 \leq Z \leq m$. Hence $\alpha_{l+1} \ldots \alpha_{m}=\gamma_{l}$ for $1 \leq \eta \leq m$. Thus, $\alpha_{\eta} \alpha_{\eta}(x)-x \alpha_{l-1}(x)$ is in $M_{\eta-1}(x)$ for $1 \leq l \leq m$. Hence, there exist $\beta_{h l}, I \leq h \leq l, 1 \leq l \leq m$, in $R$ 
such that

$$
\alpha_{\eta} \alpha_{\eta}(x)=x \alpha_{l-1}(x)+\sum_{h=1}^{l} \beta_{h l_{h-1}}(x) \text { for } \quad 1 \leq \imath \leq m,
$$

and $\alpha_{0}(x)=\alpha_{1} \ldots \alpha_{m} \alpha_{m}$

Now, using the formulations for $\alpha_{0}(x), \ldots, \alpha_{m}(x)$ in I, it follows that $\gamma_{m}$ divides $\alpha_{0}(x), \ldots, \alpha_{m}(x)$. But $\gamma_{m}$ is in $N \cup \bar{N}$ and $\alpha_{0}(x), \ldots, \alpha_{m}(x)$ generate $A(x)$ which is primitive. Thus $\gamma_{m}=1$.

VIII (showing that $\alpha_{m} \neq I$ ). If $\alpha_{m}=1$ (thus $m>0$ ) it would follow from VII that $\alpha_{m}(x)$ is contained in the ideal generated by $\alpha_{0}(x), \ldots, \alpha_{m-1}(x)$. But then this ideal would be equal to $A(x)$, contradicting the definition of $m(A(x))=m$ in IV.

IX (showing that $\beta_{1 l}, \ldots, \beta_{2 l}$ are in $\left(\alpha_{2}\right)$ for $1 \leq l \leq m$ ). clearly this condition holds for $\alpha_{0}(x)$. Now continue by induction. Suppose that for some $r, 1 \leq r \leq m$, the $\alpha_{0}(x), \ldots, \alpha_{r_{-1}}(x)$ have been chosen as in $\mathrm{V}$ so that the coefficients $\beta_{h l}, 1 \leq h \leq l, 1 \leq 2 \leq r-1$, satisfy condition ( $i$ ii).

Let $\alpha_{p}^{*}(x)$ be any polynomial in $A(x)$ which might replace $\alpha_{p}(x)$. Then $\alpha_{r}^{*}(x)$ and $\alpha_{r}(x)$ have the same leading coefficient $\alpha_{r+1} \cdots \alpha_{m}$. Thus, since $\alpha_{r}^{*}(x)$ is in $M_{r}(x)$, there exist $\delta_{0}, \ldots, \delta_{r-1}$ in $R$ such that

$$
\alpha_{r}^{*}(x)=\alpha_{r}(x)+\delta_{r-1} \alpha_{r-1}(x)+\ldots+\delta_{0} \alpha_{0}(x) .
$$

From this it follows that

$$
\alpha_{p} \alpha_{p}^{*}(x)=x \alpha_{r-1}(x)+\sum_{l=1}^{r}\left(\beta_{l x}+\alpha_{r} \delta_{\eta-1}\right) \alpha_{\eta-1}(x)
$$

Thus $\beta_{\eta_{r}}^{*}=\beta_{\eta_{r}}+\alpha_{r} \delta_{Z_{-1}}, \quad 1 \leq Z \leq r$, and condition (iii) is satisfied. 


\section{References}

[1] Adolf Hurwitz, Vorlesungen über die Zahlentheorie der Quaternionen (Julius Springer, Berlin, 1919).

[2] L. Redéi, AZgebra, Volume 1 (International Series of Monographs in Pure and Applied Mathematics, 91. Pergamon, Oxford, London, Edinburgh, New York, Toronto, Sydney, Paris, Braunschweig; Akaaárniai Kiadó, Hungary; 1967).

[3] G. Szekeres, "A canonical basis for the ideals of a polynomial domain", Amer. Math. Monthly 59 (1952), 379-386.

Department of Mathematics,

Pennsylvania State University,

University Park,

Pennsylvania,

USA. 